\title{
Inhomogeneous poly-scale refinement type equations and Markov operators with perturbations
}

\author{
Rafał Kapica and Janusz Morawiec
}

\begin{abstract}
Given measure spaces $\left(\Omega_{1}, \mathcal{A}_{1}, \mu_{1}\right), \ldots,\left(\Omega_{N}, \mathcal{A}_{N}, \mu_{N}\right)$, functions $\varphi_{1}: \mathbb{R}^{m} \times \Omega_{1} \rightarrow \mathbb{R}^{m}, \ldots, \varphi_{N}: \mathbb{R}^{m} \times \Omega_{N} \rightarrow \mathbb{R}^{m}$ and $g: \mathbb{R}^{m} \rightarrow \mathbb{R}$, we present results on the existence of solutions $f: \mathbb{R}^{m} \rightarrow \mathbb{R}$ of the inhomogeneous poly-scale refinement type equation of the form

$$
f(x)=\sum_{n=1}^{N} \int_{\Omega_{n}}\left|\operatorname{det}\left(\varphi_{n}\right)_{x}^{\prime}\left(x, \omega_{n}\right)\right| f\left(\varphi_{n}\left(x, \omega_{n}\right)\right) d \mu_{n}\left(\omega_{n}\right)+g(x)
$$

in some special classes of functions. The results are obtained by Banach fixed point theorem applied to a perturbed Markov operator connected with the considered inhomogeneous poly-scale refinement type equation.

Mathematics Subject Classification. Primary 37H99, 37N99; Secondary 39B12.

Keywords. Inhomogeneous poly-scale refinement type equations, Markov operators with perturbations, fixed points, $L^{p}$-solutions, continuous and bounded solutions, compactly supported solutions.
\end{abstract}

\section{Introduction}

Fix $m, N \in \mathbb{N}$, measure spaces $\left(\Omega_{1}, \mathcal{A}_{1}, \mu_{1}\right), \ldots,\left(\Omega_{N}, \mathcal{A}_{N}, \mu_{N}\right)$ and functions $\varphi_{1}: \mathbb{R}^{m} \times \Omega_{1} \rightarrow \mathbb{R}^{m}, \ldots, \varphi_{N}: \mathbb{R}^{m} \times \Omega_{N} \rightarrow \mathbb{R}^{m}, g: \mathbb{R}^{m} \rightarrow \mathbb{R}$. We are interested in solutions $f: \mathbb{R}^{m} \rightarrow \mathbb{R}$ of the inhomogeneous poly-scale refinement type equation

$$
f(x)=\sum_{n=1}^{N} \int_{\Omega_{n}}\left|\operatorname{det}\left(\varphi_{n}\right)_{x}^{\prime}\left(x, \omega_{n}\right)\right| f\left(\varphi_{n}\left(x, \omega_{n}\right)\right) d \mu_{n}\left(\omega_{n}\right)+g(x)
$$

in the space of all $p$ th power Lebesgue integrable functions as well as in the space of all continuous and bounded functions. If for every $n \in\{1, \ldots, N\}$ 
the function $\varphi_{n}: \mathbb{R}^{m} \times \Omega_{n} \rightarrow \mathbb{R}^{m}$ is of the form

$$
\varphi_{n}\left(x, \omega_{n}\right)=K_{n}\left(\omega_{n}\right) x-M_{n}\left(\omega_{n}\right)
$$

with given functions

$$
K_{n}: \Omega_{n} \rightarrow \mathbb{R}^{m \times m} \quad \text { and } \quad M_{n}: \Omega_{n} \rightarrow \mathbb{R}^{m},
$$

then equation (1.1) becomes the inhomogeneous poly-scale refinement type equation

$$
f(x)=\sum_{n=1}^{N} \int_{\Omega_{n}}\left|\operatorname{det} K_{n}\left(\omega_{n}\right)\right| f\left(K_{n}\left(\omega_{n}\right) x-M_{n}\left(\omega_{n}\right)\right) d \mu_{n}\left(\omega_{n}\right)+g(x) .
$$

Taking $g=0$ in (1.3) we obtain the following homogeneous poly-scale refinement type equation

$$
f(x)=\sum_{n=1}^{N} \int_{\Omega_{n}}\left|\operatorname{det} K_{n}\left(\omega_{n}\right)\right| f\left(K_{n}\left(\omega_{n}\right) x-M_{n}\left(\omega_{n}\right)\right) d \mu_{n}\left(\omega_{n}\right) .
$$

This equation extends the discrete poly-scale refinement equation which has been studied in $[5,26,27]$. If $N=1$, then equation (1.3) reduces to the inhomogeneous refinement type equation

$$
f(x)=\int_{\Omega}|\operatorname{det} K(\omega)| f(K(\omega) x-M(\omega)) d \mu(\omega)+g(x) .
$$

Inhomogeneous refinement equations are motivated by constructions of multiwavelets, multichannel filters and constructions of wavelets on a finite interval (see, e.g., [4, 10, 23]). Various inhomogeneous forms of equation (1.4) have been investigated in $[6,12,24,25]$.

Several problems from different areas of pure and applied mathematics lead to the problem of the existence of nontrivial Lebesgue integrable solutions of refinement type equation (1.4) with $g=0$, i.e., the homogeneous refinement type equation

$$
f(x)=\int_{\Omega}|\operatorname{det} K(\omega)| f(K(\omega) x-M(\omega)) d \mu(\omega)
$$

(for more details see the survey [15] and the references therein). It turns out that in some applications, continuous and bounded or continuous and compactly supported solutions of homogeneous refinement equations are important. Such solutions have significant applications in wavelet theory, approximation theory, theory of subdivision schemes, computer graphics, physics, combinatorial number theory and many others (see, e.g., [2, 7, 8, 9, 18, 19, 22]).

From the point of view of applications, all results on the existence of "good" solutions of homogeneous as well as of inhomogeneous refinement equations are very important (see [3] where it is showed how nonexistence of "good" solutions of a refinement equation can lead to anomalous behavior of numerical methods for a construction of wavelets). Let us note that refinement equations always have plenty of "bad" solutions, even extremely strange (see, e.g., $[1,15,20]$ ). 
To find conditions under which homogeneous refinement type equation (1.5) has a nontrivial Lebesgue integrable solution is rather difficult; some results in this direction can be found in [14, 21]. To the best of our knowledge, there is no result concerning the existence of a nontrivial Lebesgue integrable solution of the homogeneous poly-scale refinement type equation

$$
f(x)=\sum_{n=1}^{N} \int_{\Omega_{n}}\left|\operatorname{det}\left(\varphi_{n}\right)_{x}^{\prime}\left(x, \omega_{n}\right)\right| f\left(\varphi_{n}\left(x, \omega_{n}\right)\right) d \mu_{n}\left(\omega_{n}\right) .
$$

Nevertheless, it is possible to formulate conditions under which homogeneous poly-scale refinement type equation (1.6) has no nontrivial Lebesgue integrable solution (see [13] for the case where $N=1$ ). Therefore, if homogeneous poly-scale refinement type equation (1.6) has no nontrivial Lebesgue integrable solution, we can ask if its inhomogeneous counterpart (1.1), obtained by adding to the right-hand side of (1.6) a perturbation function $g$, has such a solution.

If $\mathcal{F}$ is a given class of functions, then the existence of a solution $f \in \mathcal{F}$ of inhomogeneous poly-scale refinement type equation (1.1) is a consequence of the existence of a fixed point of the operator $P: \mathcal{F} \rightarrow \mathcal{F}$ given by

$$
P f=\sum_{n=1}^{N} \int_{\Omega_{n}}\left|\operatorname{det}\left(\varphi_{n}\right)_{x}^{\prime}\left(\cdot, \omega_{n}\right)\right| f\left(\varphi_{n}\left(\cdot, \omega_{n}\right)\right) d \mu_{n}\left(\omega_{n}\right)+g .
$$

It turns out that the operator $P$ happens (under suitable assumptions) to be the Markov operator in the case where $g=0$; for the definition of the Markov operator and more information on it see [17]. Asymptotically stability of Markov operators has been explored in [21] to study the problem of the existence of nontrivial Lebesgue integrable solutions of homogeneous refinement type equation (1.5). In this paper, we are going to examine the Banach fixed point theorem to obtain results on the existence of a $p$ th power Lebesgue integrable solution as well as a continuous and bounded solution of equation (1.1) and of its special case (1.3). Thus we are looking for conditions, on the spaces $\left(\Omega_{1}, \mathcal{A}_{1}, \mu_{1}\right), \ldots,\left(\Omega_{N}, \mathcal{A}_{N}, \mu_{N}\right)$ and the given functions $\varphi_{1}, \ldots, \varphi_{N}$ and $g$, guaranteeing that the operator $P$ is well defined and satisfies assumptions of the Banach fixed point theorem.

\section{Notation}

Given a real number $p \geq 1$, we write $L^{p}$ to denote the Banach space of all $p$ th power Lebesgue measurable functions $f: \mathbb{R}^{m} \rightarrow \mathbb{R}$ with the standard norm

$$
\|f\|_{p}=\left(\int_{\mathbb{R}^{m}}|f(x)|^{p} d x\right)^{1 / p}
$$

If $f \in L^{p}$, then $f=f_{+}-f_{-}$, where $f_{+}$and $f_{-}$are the positive and negative parts of $f$; i.e., $f_{+}=\max \{f, 0\}$ and $f_{-}=\max \{-, 0\}$. We say that a point $x \in \mathbb{R}^{m}$ is in the support of $f \in L^{p}$ if for every neighborhood $U_{x} \subset \mathbb{R}^{m}$ of $x$ 
we have

$$
l_{m}\left(\left\{y \in U_{x}: f(y) \neq 0\right\}\right)>0 ;
$$

here and throughout, $l_{m}$ denotes the Lebesgue measure on $\mathbb{R}^{m}$. Given $f \in L^{p}$, it is easy to see that the set

$$
\operatorname{supp} f=\left\{x \in \mathbb{R}^{m}: x \text { is in the support of } f\right\}
$$

is closed; it is called the support of $f$ and if it is compact we say that $f$ is compactly supported.

We write $C_{B}$ to denote the Banach space of all continuous and bounded functions $f: \mathbb{R}^{m} \rightarrow \mathbb{R}$ with the supremum norm

$$
\|f\|_{\text {sup }}=\sup \left\{|f(x)|: x \in \mathbb{R}^{m}\right\} \text {. }
$$

Since $\mathbb{R}^{m}$ is a noncompact space, we can consider the subspace $C_{C} \subset C_{B}$ of all functions $f: \mathbb{R}^{m} \rightarrow \mathbb{R}$ with compact support; in contrast to the definition of the support of $p$ th power Lebesgue measurable functions, we say that a continuous function $f: \mathbb{R} \rightarrow \mathbb{R}$ has a compact support if the set

$$
\operatorname{supp} f=\operatorname{cl}\left\{x \in \mathbb{R}^{m}: f(x) \neq 0\right\},
$$

called the support of $f$, is compact. The space $C_{C}$ endowed in the supremum norm is not, in general, complete. The completion of $C_{C}$ is the space $C_{0}$ consisting of all those continuous functions $f: \mathbb{R}^{m} \rightarrow \mathbb{R}$ that vanish at infinity; i.e., $f \in C_{0}$ if and only if $f \in C_{B}$ and the set $\operatorname{cl}\left\{x \in \mathbb{R}^{m}:|f(x)| \geq \varepsilon\right\}$ is compact for every $\varepsilon>0$. It is easy to prove that $f \in C_{0}$ if and only if $f$ is continuous and $\lim _{\|x\| \rightarrow+\infty} f(x)=0$, where $\|\cdot\|$ is an arbitrary norm in $\mathbb{R}^{m}$.

The symbol $\mathcal{B}$ will denote the family of all Borel subsets of $\mathbb{R}^{m}$.

From now on we assume that $\left(\Omega_{1}, \mathcal{A}_{1}, \mu_{1}\right), \ldots,\left(\Omega_{N}, \mathcal{A}_{N}, \mu_{N}\right)$ are complete finite measure spaces, and $P$ denotes the operator given by (1.7).

\section{3. $L^{1}$-solutions}

Throughout this section we assume that $g \in L^{1}$.

As it was mentioned earlier, our aim is to use the Banach fixed point theorem to the operator $P$ defined by (1.7). For this purpose we need to know that $P$ transforms the space $L^{1}$ into itself. Before we give conditions on $\varphi_{n}$ 's under which $P\left(L^{1}\right) \subset L^{1}$, we recall that $f \in L^{1}$ is called an $L^{1}$-solution of equation (1.1), if every representative of $f$ satisfies (1.1) for almost all $x \in \mathbb{R}^{m}$ with respect to $l_{m}$.

Lemma 3.1. Assume that for every $n \in\{1, \ldots, N\}$ the function

$$
\varphi_{n}: \mathbb{R}^{m} \times \Omega_{n} \rightarrow \mathbb{R}^{m}
$$

satisfies the following conditions:

(3.1) $\varphi_{n}\left(\cdot, \omega_{n}\right)$ is a diffeomorphism from $\mathbb{R}^{m}$ onto $\mathbb{R}^{m}$ for every $\omega_{n} \in \Omega_{n}$;

(3.2) $\varphi_{n}(x, \cdot)$ is $\mathcal{A}_{n}$-measurable for every $x \in \mathbb{R}^{m}$;

(3.3) $\left(l_{m} \otimes \mu_{n}\right)\left(\varphi_{n}^{-1}(B)\right)=0$ for every Borel set $B \subset \mathbb{R}^{m}$ with $l_{m}(B)=0$. 


\section{Then}

(i) if $f: \mathbb{R}^{m} \rightarrow \mathbb{R}$ is a Lebesgue integrable function, then so is the function

$$
\mathbb{R}^{m} \ni x \longmapsto \sum_{n=1}^{N} \int_{\Omega_{n}}\left|\operatorname{det}\left(\varphi_{n}\right)_{x}^{\prime}\left(x, \omega_{n}\right)\right| f\left(\varphi_{n}\left(x, \omega_{n}\right)\right) d \mu_{n}\left(\omega_{n}\right) ;
$$

(ii) if $f, \tilde{f}: \mathbb{R}^{m} \rightarrow \mathbb{R}$ are Lebesgue integrable functions such that $f(x)=\tilde{f}(x)$ for almost all $x \in \mathbb{R}^{m}$ and $f$ satisfies equation (1.1) for almost all $x \in$ $\mathbb{R}^{m}$, then also $\tilde{f}$ satisfies equation (1.1) for almost all $x \in \mathbb{R}^{m}$;

(iii) we have $P\left(L^{1}\right) \subset L^{1}$.

Proof. (i) Fix $n \in\{1, \ldots, N\}$.

Conditions (3.1) and (3.2) imply that both functions $\varphi_{n}$ and $\operatorname{det}\left(\varphi_{n}\right)_{x}^{\prime}$ are $\mathcal{B} \otimes \mathcal{A}_{n}$-measurable (see [11] or [16]).

Fix a Lebesgue integrable function $f: \mathbb{R}^{m} \rightarrow[0,+\infty)$ and a set $B \in \mathcal{B}$. From condition (3.3) we obtain that the set $\left(f \circ \varphi_{n}\right)^{-1}(B)$ belongs to the completion $\overline{\mathcal{B} \otimes \mathcal{A}_{n}}$ of $\mathcal{B} \otimes \mathcal{A}_{n}$. Consequently, the function $\left|\operatorname{det}\left(\varphi_{n}\right)_{x}^{\prime}\right|\left(f \circ \varphi_{n}\right)$ is $\overline{\mathcal{L}_{m} \otimes \mathcal{A}_{n}}$-measurable and

$$
\begin{array}{r}
\int_{\mathbb{R}^{m} \times \Omega_{n}}\left|\operatorname{det}\left(\varphi_{n}\right)_{x}^{\prime}\left(x, \omega_{n}\right)\right| f\left(\varphi_{n}\left(x, \omega_{n}\right)\right) d\left(\overline{l_{m} \otimes \mu_{n}}\right)\left(x, \omega_{n}\right) \\
=\mu_{n}\left(\Omega_{n}\right) \int_{\mathbb{R}^{m}} f(x) d x=\mu_{n}\left(\Omega_{n}\right)\|f\|_{1}<+\infty .
\end{array}
$$

Since $\mathcal{A}_{n}$ is complete, we conclude from the Fubini theorem that the function

$$
\left|\operatorname{det}\left(\varphi_{n}\right)_{x}^{\prime}(x, \cdot)\right|\left(f \circ \varphi_{n}\right)(x, \cdot)
$$

is $\mu_{n}$-integrable for almost all $x \in \mathbb{R}^{m}$ and that the function

$$
\int_{\Omega_{n}}\left|\operatorname{det}\left(\varphi_{n}\right)_{x}^{\prime}\left(\cdot, \omega_{n}\right)\right| f\left(\varphi_{n}\left(\cdot, \omega_{n}\right)\right) d \mu_{n}\left(\omega_{n}\right)
$$

is Lebesgue integrable.

Fix now an arbitrary Lebesgue integrable function $f: \mathbb{R}^{m} \rightarrow \mathbb{R}$. Since both the functions $f_{+}$and $f_{-}$are nonnegative and Lebesgue integrable, we conclude that both the functions

$$
\int_{\Omega_{n}}\left|\operatorname{det}\left(\varphi_{n}\right)_{x}^{\prime}\left(\cdot, \omega_{n}\right)\right| f_{+}\left(\varphi_{n}\left(\cdot, \omega_{n}\right)\right) d \mu_{n}\left(\omega_{n}\right)
$$

and

$$
\int_{\Omega_{n}}\left|\operatorname{det}\left(\varphi_{n}\right)_{x}^{\prime}\left(\cdot, \omega_{n}\right)\right| f_{-}\left(\varphi_{n}\left(\cdot, \omega_{n}\right)\right) d \mu_{n}\left(\omega_{n}\right)
$$

are Lebesgue integrable. In consequence, the function

$$
\int_{\Omega_{n}}\left|\operatorname{det}\left(\varphi_{n}\right)_{x}^{\prime}\left(\cdot, \omega_{n}\right)\right| f\left(\varphi_{n}\left(\cdot, \omega_{n}\right)\right) d \mu_{n}\left(\omega_{n}\right)
$$

is also Lebesgue integrable.

Finally, the function given by (3.4) is Lebesgue integrable, because it is a finite sum of Lebesgue integrable functions. 
(ii) Fix two Lebesgue integrable functions $f, \tilde{f}: \mathbb{R}^{m} \rightarrow \mathbb{R}$ and assume that there exists a set $B \in \mathcal{B}$ with $l_{m}(B)=0$ such that $f(x)=\tilde{f}(x)$ for every $x \notin B$. Since the right-hand side of equation (1.1) is a Lebesgue integrable function of variable $x$ by assertion (i), we can assume that (1.1) holds for every $x \notin A$ with $l_{m}(A)=0$. By (3.3) for every $n \in\{1, \ldots, N\}$ we have

$$
0=\left(l_{m} \otimes \mu_{n}\right)\left(\varphi_{n}^{-1}(B)\right)=\int_{\mathbb{R}^{m}} \mu\left(\varphi_{n}^{-1}(B)_{x}\right) d x .
$$

Then for every $n \in\{1, \ldots, N\}$ there exists a Lebesgue measurable set $C_{n}$ such that $l_{m}\left(C_{n}\right)=0$ and $\mu_{n}\left(\varphi_{n}^{-1}(B)_{x}\right)=0$ for every $x \notin C_{n}$. Hence, if

$$
x \notin A \cup B \cup \bigcup_{n=1}^{N} C_{n},
$$

then

$$
\begin{aligned}
\tilde{f}(x) & =f(x) \\
& =\sum_{n=1}^{N} \int_{\Omega_{n}}\left|\operatorname{det}\left(\varphi_{n}\right)_{x}^{\prime}\left(x, \omega_{n}\right)\right| f\left(\varphi_{n}\left(x, \omega_{n}\right)\right) d \mu_{n}\left(\omega_{n}\right)+g(x) \\
& =\sum_{n=1}^{N} \int_{\Omega_{n} \backslash \varphi_{n}^{-1}(B)_{x}}\left|\operatorname{det}\left(\varphi_{n}\right)_{x}^{\prime}\left(x, \omega_{n}\right)\right| f\left(\varphi_{n}\left(x, \omega_{n}\right)\right) d \mu_{n}\left(\omega_{n}\right)+g(x) \\
& =\int_{\Omega_{n} \backslash \varphi_{n}^{-1}(B)_{x}}\left|\operatorname{det}\left(\varphi_{n}\right)_{x}^{\prime}\left(x, \omega_{n}\right)\right| \tilde{f}\left(\varphi_{n}\left(x, \omega_{n}\right)\right) d \mu_{n}\left(\omega_{n}\right)+g(x) \\
& =\sum_{n=1}^{N} \int_{\Omega_{n}}\left|\operatorname{det}\left(\varphi_{n}\right)_{x}^{\prime}\left(x, \omega_{n}\right)\right| \tilde{f}\left(\varphi_{n}\left(x, \omega_{n}\right)\right) d \mu_{n}\left(\omega_{n}\right)+g(x) ;
\end{aligned}
$$

i.e., $\tilde{f}$ satisfies (1.1) for almost all $x \in \mathbb{R}^{m}$.

(iii) Fix $f \in L^{1}$ and choose two representatives $f_{1}$ and $f_{2}$ of $f$. By assertion (i) we infer that both the functions $P f_{1}$ and $P f_{2}$ are Lebesgue integrable. Now by the same arguments as in the proof of assertion (ii) we conclude that $P f_{1}(x)=P f_{2}(x)$ for almost all $x \in \mathbb{R}^{m}$.

Observe that assertion (ii) of the above lemma says that the definition of $L^{1}$-solutions of equation (1.1) is well posed.

In the case where the functions $\varphi_{n}$ 's are of the form (1.2) we do not need any special condition guaranteeing that $P: L^{1} \rightarrow L^{1}$. More precisely, we have the following observation.

Remark 3.2. Let $K_{n}: \Omega_{n} \rightarrow \mathbb{R}^{m \times m}$ and $M_{n}: \Omega_{n} \rightarrow \mathbb{R}^{m}$ be Lebesgue measurable functions. If $\operatorname{det} K_{n}(\omega) \neq 0$ for every $\omega_{n} \in \Omega_{n}$, then the function $\varphi_{n}: \mathbb{R}^{m} \times \Omega_{n} \rightarrow \mathbb{R}^{m}$ given by (1.2) satisfies conditions (3.1)-(3.3).

Now we are in a position to formulate our first result on the existence of a unique $L^{1}$-solution of equation (1.1) in the space $L^{1}$.

Theorem 3.3. Assume that for every $n \in\{1, \ldots, N\}$ the function

$$
\varphi_{n}: \mathbb{R}^{m} \times \Omega_{n} \rightarrow \mathbb{R}^{m}
$$


satisfies conditions (3.1)-(3.3). If $\sum_{n=1}^{N} \mu\left(\Omega_{n}\right)<1$, then equation (1.1) has exactly one $L^{1}$-solution $f$ and for every $f_{0} \in L^{1}$ the sequence $\left(P^{n} f_{0}\right)_{n \in \mathbb{N}}$ converges to $f$ in $L^{1}$. Moreover,

(i) if $g$ is of constant sign, then so is $f$;

(ii) if $\int_{\mathbb{R}^{m}} g(x) d x=0$, then $\int_{\mathbb{R}^{m}} f(x) d x=0$;

(iii) if there exists a closed set $Z \subset \mathbb{R}^{m}$ such that

$$
\varphi_{n}\left(Z \times \Omega_{n}\right) \subset Z \quad \text { for every } n \in\{1, \ldots, N\}
$$

and if $\operatorname{supp} g \subset Z$, then $\operatorname{supp} f \subset Z$; in particular, if $Z$ is compact, then $f$ is compactly supported.

Proof. By Lemma 3.1 we have $P: L^{1} \rightarrow L^{1}$. Moreover, for all $f_{1}, f_{2} \in L^{1}$ we obtain

$$
\left\|P f_{1}-P f_{2}\right\|_{1} \leq \sum_{n=1}^{N} \mu_{n}\left(\Omega_{n}\right)\left\|f_{1}-f_{2}\right\|_{1} .
$$

The Banach fixed point theorem completes the proof of the main part of the theorem.

(i) Clearly, both the sets

$$
L_{+}^{1}=\left\{h \in L^{1}: h \geq 0\right\} \quad \text { and } \quad L_{-}^{1}=\left\{h \in L^{1}: h \leq 0\right\}
$$

are closed subspaces of $L^{1}$. Now it is enough to observe that $P: L_{+}^{1} \rightarrow L_{+}^{1}$ and $P: L_{-}^{1} \rightarrow L_{-}^{1}$.

(ii) Observe first that the set $\left\{h \in L^{1}: \int_{\mathbb{R}^{m}} h(x) d x=0\right\}$ is a closed subspace of $L^{1}$.

Taking the Fourier transform of both sides of equation (1.1), we obtain

$$
\begin{aligned}
\widehat{f}(y) & =\int_{\mathbb{R}^{m}} e^{i x y} f(x) d x \\
& =\sum_{n=1}^{N} \int_{\Omega_{n}} \int_{\mathbb{R}^{m}} e^{i x y}\left|\operatorname{det}\left(\varphi_{n}\right)_{x}^{\prime}\left(x, \omega_{n}\right)\right| f\left(\varphi_{n}\left(x, \omega_{n}\right)\right) d x d \mu_{n}\left(\omega_{n}\right)+\widehat{g}(y)
\end{aligned}
$$

for every $y \in \mathbb{R}$. Hence for $y=0$ we get

$$
\left[1-\sum_{n=1}^{N} \mu_{n}\left(\Omega_{n}\right)\right] \widehat{f}(0)=\widehat{g}(0)=\int_{\mathbb{R}^{m}} g(x) d x=0,
$$

and in consequence,

$$
\int_{\mathbb{R}^{m}} f(x) d x=\widehat{f}(0)=0 .
$$

(iii) Since $Z$ is closed, it follows that

$$
X=\left\{h \in L^{1}: \operatorname{supp} h \subset Z\right\}
$$

is a closed subspace of $L^{1}$.

Fix $h \in X$. By $(3.5)$ we have $\operatorname{supp}\left(h \circ \varphi_{n}\right) \subset Z$ for every $n \in\{1, \ldots, N\}$. This jointly with supp $g \subset Z$ implies supp $P h \subset Z$ and, in consequence, we obtain $P: X \rightarrow X$. 
Corollary 3.4. Assume that for every $n \in\{1, \ldots, N\}$ the functions

$$
K_{n}: \Omega_{n} \rightarrow \mathbb{R}^{m \times m} \quad \text { and } \quad M_{n}: \Omega_{n} \rightarrow \mathbb{R}^{m}
$$

are Lebesgue measurable. If $\sum_{n=1}^{N} \mu\left(K_{n} \neq 0\right)<1$, then equation (1.3) has exactly one $L^{1}$-solution $f$ and for every $f_{0} \in L^{1}$ the sequence $\left(P^{n} f_{0}\right)_{n \in \mathbb{N}}$ converges to $f$ in $L^{1}$. Moreover,

(i) if $g$ is of constant sign, then so is $f$;

(ii) if $\int_{\mathbb{R}^{m}} g(x) d x=0$, then $\int_{\mathbb{R}^{m}} f(x) d x=0$;

(ii) if there exists a closed set $Z \subset \mathbb{R}^{m}$ such that

$$
K\left(\omega_{n}\right) Z \subset Z+M\left(\omega_{n}\right) \quad \text { for all } \omega_{n} \in \Omega_{n} \text { and } n \in\{1, \ldots, N\}
$$

and if supp $g \subset Z$, then $\operatorname{supp} f \subset Z$; in particular, if $Z$ is compact, then $f$ is compactly supported.

Proof. Putting

$$
\widetilde{\Omega}_{n}=\left\{\omega_{n} \in \Omega_{n}: \operatorname{det} K_{n}\left(\omega_{n}\right) \neq 0\right\}
$$

for every $n \in\{1, \ldots, N\}$ we get $\sum_{n=1}^{N} \mu_{n}\left(\widetilde{\Omega}_{n}\right)<1$. Now it is enough to apply Remark 3.2 and Theorem 3.3.

In the case where $g=0$, Theorem 3.3 says that the trivial function is the only $L^{1}$-solution of equation (1.6). To see it in another way suppose that, on the contrary, $f$ is a nontrivial $L^{1}$-solution of equation (1.6). Then

$$
\begin{aligned}
\|f\|_{1} & \leq \sum_{n=1}^{N} \int_{\Omega_{n}} \int_{\mathbb{R}^{m}}\left|\operatorname{det}\left(\varphi_{n}\right)_{x}^{\prime}\left(x, \omega_{n}\right) f\left(\varphi_{n}\left(x, \omega_{n}\right)\right)\right| d x d \mu_{n}\left(\omega_{n}\right) \\
& =\|f\|_{1} \sum_{n=1}^{N} \mu_{n}\left(\Omega_{n}\right)<\|f\|_{1},
\end{aligned}
$$

which is impossible.

\section{4. $L^{p}$-solutions}

Throughout this section we assume that $g \in L^{p}$ with $p>1$.

Recall that $f \in L^{p}$ is called an $L^{p}$-solution of equation (1.1), if every representative of $f$ satisfies (1.1) for almost all $x \in \mathbb{R}^{m}$ with respect to $l_{m}$.

We begin with a counterpart of Lemma 3.1 for the space $L^{p}$.

Lemma 4.1. Assume that for every $n \in\{1, \ldots, N\}$ the function

$$
\varphi_{n}: \mathbb{R}^{m} \times \Omega_{n} \rightarrow \mathbb{R}^{m}
$$

satisfies conditions (3.1)-(3.3) and

$$
\sup _{x \in \mathbb{R}^{m}}\left|\operatorname{det}\left(\varphi_{n}\right)_{x}^{\prime}(x, \cdot)\right|^{p-1} \leq\left(\Phi_{n}\right)^{p} \quad \text { with } \Phi_{n} \in L^{1}\left(\Omega_{n}\right) \text {. }
$$

Then

(i) if $f: \mathbb{R}^{m} \rightarrow \mathbb{R}$ is a pth power Lebesgue integrable function, then so is the function given by formula (3.4); 
(ii) if $f, \tilde{f}: \mathbb{R}^{m} \rightarrow \mathbb{R}$ are pth power Lebesgue integrable functions such that $f(x)=\tilde{f}(x)$ for almost all $x \in \mathbb{R}^{m}$ and $f$ satisfies equation (1.1) for almost all $x \in \mathbb{R}^{m}$, then also $\tilde{f}$ satisfies equation (1.1) for almost all $x \in \mathbb{R}^{m}$;

(iii) we have $P\left(L^{p}\right) \subset L^{p}$.

Proof. (i) Fix $n \in\{1, \ldots, N\}$ and $p$ th power Lebesgue integrable function $f: \mathbb{R}^{m} \rightarrow \mathbb{R}$. In the same way as in the proof of assertion (i) of Lemma 3.1, conditions (3.1)-(3.3) imply that the function

$$
\left|\operatorname{det}\left(\varphi_{n}\right)_{x}^{\prime}\right|\left(f \circ \varphi_{n}\right)
$$

is $\overline{\mathcal{L}_{m} \otimes \mathcal{A}_{n}}$-measurable. By the Minkowski inequality for integrals and (4.1) we have

$$
\begin{aligned}
& \left(\int_{\mathbb{R}^{m}}\left[\int_{\Omega_{n}}\left|\operatorname{det}\left(\varphi_{n}\right)_{x}^{\prime}\left(x, \omega_{n}\right)\right| f_{+}\left(\varphi_{n}\left(x, \omega_{n}\right)\right) d \mu_{n}\left(\omega_{n}\right)\right]^{p} d x\right)^{1 / p} \\
& \quad \leq \int_{\Omega_{n}}\left(\int_{\mathbb{R}^{m}}\left[\left|\operatorname{det}\left(\varphi_{n}\right)_{x}^{\prime}\left(x, \omega_{n}\right)\right| f_{+}\left(\varphi_{n}\left(x, \omega_{n}\right)\right)\right]^{p} d x\right)^{1 / p} d \mu_{n}\left(\omega_{n}\right) \\
& \leq \int_{\Omega_{n}}\left(\int_{\mathbb{R}^{m}}\left[\Phi_{n}\left(\omega_{n}\right) f_{+}(y)\right]^{p} d y\right)^{1 / p} d \mu_{n}(\omega) \\
& \quad=\left\|\Phi_{n}\right\|_{1}\left\|f_{+}\right\|_{p}<+\infty,
\end{aligned}
$$

which shows that the function

$$
\int_{\Omega_{n}}\left|\operatorname{det}\left(\varphi_{n}\right)_{x}^{\prime}\left(\cdot, \omega_{n}\right)\right| f_{+}\left(\varphi_{n}\left(\cdot, \omega_{n}\right)\right) d \mu_{n}\left(\omega_{n}\right)
$$

is $p$ th power Lebesgue integrable. In a similar way we can prove that the function

$$
\int_{\Omega_{n}}\left|\operatorname{det}\left(\varphi_{n}\right)_{x}^{\prime}\left(\cdot, \omega_{n}\right)\right| f_{-}\left(\varphi_{n}\left(\cdot, \omega_{n}\right)\right) d \mu_{n}\left(\omega_{n}\right)
$$

is $p$ th power Lebesgue integrable. Consequently, the function

$$
\int_{\Omega_{n}}\left|\operatorname{det}\left(\varphi_{n}\right)_{x}^{\prime}\left(\cdot, \omega_{n}\right)\right| f\left(\varphi_{n}\left(\cdot, \omega_{n}\right)\right) d \mu_{n}\left(\omega_{n}\right)
$$

is $p$ th power Lebesgue integrable. Finally, the function given by (3.4) is $p$ th power Lebesgue integrable, because it is a finite sum of $p$ th power Lebesgue integrable functions.

Assertions (ii) and (iii) can be proved in the same manner as assertions (ii) and (iii) of Lemma 3.1.

In the case where the functions $\varphi_{n}$ 's are of the form (1.2) we need a condition on the functions $K_{n}$ 's guaranteeing that (4.1) holds.

Remark 4.2. Let $K_{n}: \Omega_{n} \rightarrow \mathbb{R}^{m \times m}$ and $M_{n}: \Omega_{n} \rightarrow \mathbb{R}^{m}$ be Lebesgue measurable functions. If

$$
\left(\operatorname{det} K_{n}\right)^{(p-1) / p} \in L^{1}\left(\Omega_{n}\right),
$$

then the function $\varphi_{n}: \mathbb{R}^{m} \times \Omega_{n} \rightarrow \mathbb{R}^{m}$ given by (1.2) satisfies condition (4.1). 
Now we can formulate a result on the existence of the unique $L^{p}$-solution of equation (1.1) in the space $L^{p}$.

Theorem 4.3. Assume that for every $n \in\{1, \ldots, N\}$ the function

$$
\varphi_{n}: \mathbb{R}^{m} \times \Omega_{n} \rightarrow \mathbb{R}^{m}
$$

satisfies conditions (3.1)-(3.3) and (4.1). If $\sum_{n=1}^{N}\left\|\Phi_{n}\right\|_{1}<1$, then equation (1.1) has exactly one $L^{p}$-solution $f$ and for every $f_{0} \in L^{p}$ the sequence $\left(P^{n} f_{0}\right)_{n \in \mathbb{N}}$ converges to $f$ in $L^{p}$. Moreover,

(i) if $g$ is of constant sign, then so is $f$;

(ii) if there exists a closed set $Z \subset \mathbb{R}^{m}$ such that (3.5) holds and if $\operatorname{supp} g \subset$ $Z$, then $\operatorname{supp} f \subset Z$; in particular, if $Z$ is compact, then $f$ is compactly supported.

Proof. By Lemma 4.1 we have $P: L^{p} \rightarrow L^{p}$. Fix $f_{1}, f_{2} \in L^{p}$. Then the Minkowski inequality for integrals and condition (4.1) imply that

$$
\begin{aligned}
& \left\|P f_{1}-P f_{2}\right\|_{p} \\
& \leq\left(\int _ { \mathbb { R } ^ { m } } \left[\sum_{n=1}^{N} \int_{\Omega_{n}} \mid \operatorname{det}\left(\varphi_{n}\right)_{x}^{\prime}\left(x, \omega_{n}\right)\right.\right. \\
& \left.\left.\quad \times\left[f_{1}\left(\varphi_{n}\left(x, \omega_{n}\right)\right)-f_{2}\left(\varphi_{n}\left(x, \omega_{n}\right)\right)\right] \mid d \mu_{n}\left(\omega_{n}\right)\right]^{p} d x\right)^{1 / p} \\
& \leq \sum_{n=1}^{N}\left(\int _ { \mathbb { R } ^ { m } } \left[\int_{\Omega_{n}} \mid \operatorname{det}\left(\varphi_{n}\right)_{x}^{\prime}\left(x, \omega_{n}\right)\right.\right. \\
& \leq \sum_{n=1}^{N} \int_{\Omega_{n}}\left(\int_{\mathbb{R}^{m}} \mid \operatorname{det}\left(\varphi_{n}\right)_{x}^{\prime}\left(x, \omega_{n}\right)\right. \\
& \left.\left.\times\left[f_{1}\left(\varphi_{n}\left(x, \omega_{n}\right)\right)-f_{2}\left(\varphi_{n}\left(x, \omega_{n}\right)\right)\right] \mid d \mu_{n}\left(\omega_{n}\right)\right]^{p} d x\right)^{1 / p} \\
& \leq \sum_{n=1}^{N} \int_{\Omega_{n}}\left(\int_{\mathbb{R}^{m}}\left[\Phi_{n}\left(\omega_{n}\right)\right]^{p}\left|f_{1}(y)-f_{2}(y)\right|^{p} d y\right)^{1 / p} d \mu_{n}\left(\omega_{n}\right) \\
& =\sum_{n=1}^{N}\left\|\Phi_{n}\right\|_{1}\left\|f_{1}-f_{2}\right\|_{p} .
\end{aligned}
$$

The Banach fixed point theorem completes the proof of the main part of the theorem.

Assertions (i) and (ii) hold because all the sets $L_{+}^{p}=\left\{h \in L^{p}: h \geq 0\right\}, L_{-}^{p}=\left\{h \in L^{p}: h \leq 0\right\}, X=\left\{h \in L^{p}: \operatorname{supp} h \subset Z\right\}$ are closed subspaces of $L^{p}$ and $P\left(L_{+}^{p}\right) \subset L_{+}^{p}, P\left(L_{-}^{p}\right) \subset L_{-}^{p}, P(X) \subset X$. 
An immediate consequence of Theorem 4.3 and Remarks 3.2 and 4.2 is the following corollary.

Corollary 4.4. Assume that for every $n \in\{1, \ldots, N\}$ the functions

$$
K_{n}: \Omega_{n} \rightarrow \mathbb{R}^{m \times m} \text { and } M_{n}: \Omega_{n} \rightarrow \mathbb{R}^{m}
$$

are Lebesgue measurable with $\left(\operatorname{det} K_{n}\right)^{(p-1) / p} \in L^{1}\left(\Omega_{n}\right)$. If

$$
\sum_{n=1}^{N}\left\|\left(\operatorname{det} K_{n}\right)^{(p-1) / p}\right\|_{1}<1
$$

then equation (1.3) has exactly one $L^{p}$-solution $f$ and for every $f_{0} \in L^{1}$ the sequence $\left(P^{n} f_{0}\right)_{n \in \mathbb{N}}$ converges to $f$ in $L^{1}$. Moreover,

(i) if $g$ is of constant sign, then so is $f$;

(ii) if there exists a closed set $Z \subset \mathbb{R}^{m}$ such that (3.6) holds and if $\operatorname{supp} g \subset$ $Z$, then supp $f \subset Z$; in particular, if $Z$ is compact, then $f$ is compactly supported.

\section{Continuous and bounded solutions}

Throughout this section we assume that $g \in C_{B}$.

In contrast to the previous section we recall that $f \in C_{B}$ is called solution of equation (1.1), if (1.1) holds for every $x \in \mathbb{R}^{m}$.

We begin with conditions on $\varphi_{n}$ 's under which $P: C_{B} \rightarrow C_{B}$.

Lemma 5.1. Assume that for every $n \in\{1, \ldots, N\}$ the function

$$
\varphi_{n}: \mathbb{R}^{m} \times \Omega_{n} \rightarrow \mathbb{R}^{m}
$$

satisfies conditions (3.1)-(3.3) and

$$
\sup _{x \in \mathbb{R}^{m}}\left|\operatorname{det}\left(\varphi_{n}\right)_{x}^{\prime}(x, \cdot)\right| \leq \Psi_{n} \quad \text { with } \Psi_{n} \in L^{1}\left(\Omega_{n}\right) .
$$

Then $P\left(C_{B}\right) \subset C_{B}$.

Proof. Fix a function $f \in C_{B}$. Conditions (3.1)-(3.3) imply that the function

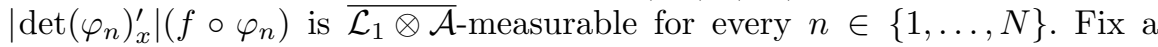
sequence $\left(x_{k}\right)_{k \in \mathbb{N}}$ of reals convergent to a real number $x$. From (3.1) we have

$$
\lim _{k \rightarrow \infty} \operatorname{det}\left(\varphi_{n}\right)_{x}^{\prime}\left(x_{k}, \omega_{n}\right) f\left(\varphi_{n}\left(x_{k}, \omega_{n}\right)\right)=\operatorname{det}\left(\varphi_{n}\right)_{x}^{\prime}\left(x, \omega_{n}\right) f\left(\varphi_{n}\left(x, \omega_{n}\right)\right),
$$

and by (5.1) we obtain

$$
\left|\operatorname{det}\left(\varphi_{n}\right)_{x}^{\prime}\left(x_{k}, \omega_{n}\right) f\left(\varphi_{n}\left(x_{k}, \omega_{n}\right)\right)\right| \leq\|f\|_{\sup } \Psi_{n}\left(\omega_{n}\right)
$$

for all $\omega_{n} \in \Omega_{n}$ and $n \in\{1, \ldots, N\}$. Hence

$$
|P f(y)| \leq\|f\|_{\text {sup }} \sum_{n=1}^{N}\left\|\Psi_{n}\right\|_{1}+\|g\|_{\text {sup }}
$$

for every $y \in \mathbb{R}^{m}$ and the Lebesgue dominated convergence theorem implies that $\lim _{k \rightarrow \infty} P f\left(x_{k}\right)=\operatorname{Pf}(x)$. In consequence, $P f \in C_{B}$. 
In the case where the functions $\varphi_{n}$ 's are of the form (1.2), we need a condition on the functions $K_{n}$ 's guaranteeing that (5.1) holds.

Remark 5.2. Let $K_{n}: \Omega_{n} \rightarrow \mathbb{R}^{m \times m}$ and $M_{n}: \Omega_{n} \rightarrow \mathbb{R}^{m}$ be Lebesgue measurable functions. If $\operatorname{det} K_{n} \in L^{1}\left(\Omega_{n}\right)$, then the function $\varphi_{n}: \mathbb{R}^{m} \times \Omega_{n} \rightarrow \mathbb{R}^{m}$ given by (1.2) satisfies condition (5.1).

Now we can formulate a result on the existence of a unique solution of equation (1.1) in the space $C_{B}$.

Theorem 5.3. Assume that for every $n \in\{1, \ldots, N\}$ the function

$$
\varphi_{n}: \mathbb{R}^{m} \times \Omega_{n} \rightarrow \mathbb{R}^{m}
$$

satisfies conditions (3.1)-(3.3) and (5.1). If $\sum_{n=1}^{N}\left\|\Psi_{n}\right\|_{1}<1$, then equation (1.1) has exactly one solution $f \in C_{B}$ and for every $f_{0} \in C_{B}$ the sequence $\left(P^{n} f_{0}\right)_{n \in \mathbb{N}}$ converges to $f$ in the supremum norm. Moreover,

(i) if $g$ is of constant sign, then so is $f$;

(ii) if $g \in C_{0}$, then $f \in C_{0}$;

(iii) if there exists a closed set $Z \subset \mathbb{R}^{m}$ such that (3.5) holds and if $\operatorname{supp} g \subset$

$Z$, then supp $f \subset Z$; in particular, if $Z$ is compact, then $f$ is compactly supported.

Proof. By Lemma 5.1 we have $P: C_{B} \rightarrow C_{B}$. Fix $f_{1}, f_{2} \in L^{p}$. Then by (5.2) we get

$$
\left\|P f_{1}-P f_{2}\right\|_{\text {sup }} \leq \sum_{n=1}^{N}\left\|\Psi_{n}\right\|_{1}\left\|f_{1}-f_{2}\right\|_{\text {sup }} .
$$

The Banach fixed point theorem completes the proof of the main part of the theorem.

Assertions (i)-(iii) hold because all the sets

$$
\begin{gathered}
C_{B}^{+}=\left\{h \in C_{B}: h \geq 0\right\}, C_{B}^{-}=\left\{h \in C_{B}: h \leq 0\right\}, C_{0}, \\
X=\left\{h \in C_{B}: \operatorname{supp} h \subset Z\right\}
\end{gathered}
$$

are closed subspaces of $C_{B}$ and $P\left(C_{B}^{+}\right) \subset C_{B}^{+}, P\left(C_{B}^{-}\right) \subset C_{B}^{-}, P\left(C_{0}\right) \subset C_{0}$, $P(X) \subset X$. To prove that $P\left(C_{0}\right) \subset C_{0}$ take $f \in C_{0}$. By (3.1) we have

$$
\left(f \circ \varphi_{n}\right)\left(\cdot, \omega_{n}\right) \in C_{0}
$$

for every $n \in\{1, \ldots, N\}$. This jointly with (5.1) and the Lebesgue dominated convergence theorem gives

$$
\lim _{\|x\| \rightarrow+\infty} \sum_{n=1}^{N} \int_{\Omega_{n}} \Psi_{n}\left(\omega_{n}\right)\left|f\left(\varphi_{n}\left(x, \omega_{n}\right)\right)\right| d \mu_{n}\left(\omega_{n}\right)=0 .
$$

Finally, since $g \in C_{0}$, it follows that $\lim _{\|x\| \rightarrow+\infty}|\operatorname{Pf}(x)|=0$.

Theorem 5.3 can be easily reformulated to get the existence and uniqueness of solutions of equation (1.1) in the space $L^{\infty}$.

We end this paper with the following immediate consequence of Theorem 5.3 and Remarks 3.2 and 5.2. 
Corollary 5.4. Assume that for every $n \in\{1, \ldots, N\}$ the functions

$$
K_{n}: \Omega_{n} \rightarrow \mathbb{R}^{m \times m} \quad \text { and } \quad M_{n}: \Omega_{n} \rightarrow \mathbb{R}^{m}
$$

are Lebesgue measurable with $\operatorname{det} K_{n} \in L^{1}\left(\Omega_{n}\right)$. If $\sum_{n=1}^{N}\left\|\operatorname{det} K_{n}\right\|_{1}<1$, then equation (1.3) has exactly one solution $f \in C_{B}$ and for every $f_{0} \in C_{B}$ the sequence $\left(P^{n} f_{0}\right)_{n \in \mathbb{N}}$ converges to $f$ in the supremum norm. Moreover,

(i) if $g$ is of constant sign, then so is $f$;

(ii) if $g \in C_{0}$, then $f \in C_{0}$;

(iii) if there exists a closed set $Z \subset \mathbb{R}^{m}$ such that (3.6) holds and if $\operatorname{supp} g \subset$ $Z$, then $\operatorname{supp} f \subset Z$; in particular, if $Z$ is compact, then $f$ is compactly supported.

\section{Acknowledgment}

This research was supported by the University of Silesia Mathematics Department (Iterative Functional Equations and Real Analysis program).

\section{References}

[1] L. Bartłomiejczyk and J. Morawiec, Irregular scaling functions with orthogonal translations. J. Math. Anal. Appl. 319 (2006), 295-301.

[2] A. S. Cavaretta, W. Dahmen and C. A. Micchelli, Stationary subdivision. Mem. Amer. Math. Soc. 93 (1991), 1-186.

[3] J. Cnops, A scaling equation with only non-measurable orthogonal solutions. Proc. Amer. Math. Soc. 128 (2000), 1975-1979.

[4] A. Cohen, I. Daubechies and P. Vial, Wavelets on the interval and fast wavelet transforms. Appl. Comput. Harmon. Anal. 1 (1993), 54-81.

[5] S. Dekel and N. Dyn, Poly-scale refinability and subdivision. Appl. Comput. Harmon. Anal. 13 (2002), 35-62.

[6] T. B. Dinsenbacher and D. P. Hardin, Nonhomogeneous refinement equations. In: Wavelets, Multiwavelets, and their Applications (San Diego, CA, 1997), A. Aldroubi and E. Lin, eds., Contemp. Math. 216, Amer. Math. Soc., Providence, RI, 1998, 117-127.

[7] G. Derfel, N. Dyn and D. Levin, Generalized refinement equations and subdivision processes. J. Approx. Theory 80 (1995), 272-297.

[8] G. Derfel and R. Schilling, Spatially chaotic configurations and functional equations with rescaling. J. Phys. A 29 (1996), 4537-4547.

[9] A. Dubickas and Z. Xu, Refinement equations and spline functions. Adv. Comput. Math. 32 (2010), 1-23.

[10] J. S. Geronimo, D. P. Hardin and P. R. Massopust, Fractal functions and wavelet expansions based on several scaling functions. J. Approx. Theory $\mathbf{7 8}$ (1994), 373-401.

[11] C. J. Himmelberg, Measurable relations. Fund. Math. 87 (1975), 53-72.

[12] R. Q. Jia, Q. T. Jiang and Z. W. Shen, Convergence of cascade algorithms associated with nonhomogeneous refinement equations. Proc. Amer. Math. Soc. 129 (2001), 415-427. 
[13] R. Kapica and J. Morawiec, On a refinement type equation. J. Appl. Anal. 4 (2008), 251-257.

[14] R. Kapica and J. Morawiec, Refinement equations and distributional fixed points. Appl. Math. Comput. 218 (2012), 7741-7746.

[15] R. Kapica and J. Morawiec, Refinement type equations: Sources and results. In: Recent Developments in Functional Equations and Inequalities: Selected Topics, Banach Center Publications 99, Polish Acad. Sci. Inst. Math., Warsaw, 2013, 87-110.

[16] K. Kuratowski, Topology. Vol. 1, Academic Press, New York, 1966.

[17] A. Lasota and M. C. Mackey, Chaos, Fractals and Noise. Stochastic Aspects of Dynamics. Springer, New York, 1994.

[18] D.-M. Lee, J.-G. Lee and S.-H. Yoon, A construction of multiresolution analysis by integral equations. Proc. Amer. Math. Soc. 130 (2002), 3555-3563.

[19] C. A. Micchelli, Mathematical Aspects of Geometric Modeling. CBMS-NSF Regional Conf. Ser. in Appl. Math. 65, SIAM, Philadelphia, PA, 1995.

[20] J. Morawiec, On the existence of irregular solutions of the two-coefficient dilation equation. Aequationes Math. 62 (2001), 79-84.

[21] J. Morawiec and R. Kapica, Refinement equations and Feller operators. Integral Equations Operator Theory 70 (2011), 323-331.

[22] V. Yu. Protasov, On the asymptotics of the binary partition function. Mat. Zametki 76 (2004), 151-156 (in Russian).

[23] G. Strang and T. Nguyen, Wavelets and Filter Banks. Wellesley-Cambridge Press, Wellesley, MA, 1996.

[24] G. Strang and D.-X. Zhou, Inhomogeneous refinement equations. J. Fourier Anal. Appl. 4 (1998), 733-747.

[25] Q. Sun, Compactly supported distributional solutions of nonstationary nonhomogeneous refinement equations. Acta Math. Sin. (Engl. Ser.) 17 (2001), 1-14.

[26] Q. Sun, Local dual and poly-scale refinability. Proc. Amer. Math. Soc. 133 (2005), 1175-1184.

[27] S.-Z. Yang, Poly-scale refinable function and their properties. Appl. Math. Mech. (English Ed.) 27 (2006), 1687-1695.

\author{
Rafał Kapica \\ Institute of Mathematics \\ University of Silesia \\ Bankowa 14, PL-40-007 Katowice \\ Poland \\ e-mail: rkapica@math.us.edu.pl \\ Janusz Morawiec \\ Institute of Mathematics \\ University of Silesia \\ Bankowa 14, PL-40-007 Katowice \\ Poland \\ e-mail: morawiec@math.us.edu.pl
}

Open Access This article is distributed under the terms of the Creative Commons Attribution 4.0 International License (http://creativecommons.org/licenses/by/4.0/), which permits unrestricted use, distribution, and reproduction in any medium, provided you give appropriate credit to the original author(s) and the source, provide a link to the Creative Commons license, and indicate if changes were made. 